\title{
Single-shot time-frequency imaging spectroscopy using an echelon mirror
}

\author{
Hiroyuki Sakaibara, ${ }^{1}$ Yuki Ikegaya, ${ }^{1}$ Ikufumi Katayama, ${ }^{2}$ and Jun Takeda ${ }^{1, *}$ \\ ${ }^{1}$ Department of Physics, Graduate School of Engineering, Yokohama National University, Yokohama 240-8501, Japan \\ ${ }^{2}$ Interdisciplinary Research Center, Yokohama National University, Yokohama 240-8501, Japan \\ *Corresponding author: jun@ynu.ac.jp
}

Received December 19, 2011; accepted January 30, 2012;

posted February 1, 2012 (Doc. ID 160127); published March 15, 2012

\begin{abstract}
We demonstrate single-shot time-frequency imaging spectroscopy with an echelon mirror for measuring ultrashort laser pulses as well as ultrafast responses of materials using the same optical setup. The echelon mirror produces a spatially encoded time delay for the probe pulse whereby both the probe and pump pulses are focused on samples with small spot size. Using the optical Kerr gate apparatus, we successfully mapped the time-frequency images of ultrashort laser pulses and subsequently evaluated the chirp characteristics with the phase-retrieval procedure on a single-shot basis. By simply replacing the Kerr medium with samples, we could also visualize the phonon-polariton oscillations in ferroelectric $\mathrm{LiNbO}_{3}$. (c) 2012 Optical Society of America

OCIS codes: $\quad 320.7100,320.7150,320.1590,300.6500$.
\end{abstract}

Since the invention of ultrashort pulsed lasers, various kinds of spectroscopic techniques have been developed for studying ultrafast transient phenomena of materials [1]. Moreover, coherent controls of molecular and solid-state dynamics have been extensively studied using femtosecond laser spectroscopy coupled with either a pulse-shaping technique or chirped laser pulses [2-4] . To perform ultrafast measurements as well as coherent controls of transient processes in materials, the following experimental abilities are highly desired; single-shotbased detections of transient signals in time-frequency space applicable to irreversible phenomena in solids; tiny focusing of the pump and probe beams at the sample position available for small crystals; and simple, quick, and accurate characterization of the laser pulses using the same optical setup as that used for the actual ultrafast measurements.

To measure the irreversible transient signals, many techniques based on single-shot detection have been proposed to date [ $\underline{5}-10]$. In these techniques, the probe pulses are manipulated to have a spatially encoded time delay, and as a result they have the capability for singleshot measurements without scanning the optical delay. However, they still have experimental difficulties; in the case of the dual-echelon technique [5,6], for example, the spectral information cannot be obtained on a singleshot basis, and the transmitted probe pulse passing through the echelons causes a spatially dependent chirp that may distort the temporal profile. Further, in pumpprobe imaging spectroscopy $[7,8]$, a large beam cross section between the pump and probe beams is needed to produce a sufficiently long time delay, and as a result a large sample is required.

For the characterization of ultrashort laser pulses, two types of popular techniques have been developed: spectral phase interferometry for direct electric field reconstruction $[11,12]$ and frequency-resolved optical gating (FROG) [13-15]. Note that some of these techniques are useful for single-shot measurements when amplified femtosecond laser systems with sufficient output power are used. However, they cannot easily be applied to measuring the ultrafast dynamics of materials, and as a result the laser pulses are typically monitored using an optical apparatus different from that used for the actual ultrafast measurements of samples.

In this Letter, we propose and demonstrate a new scheme for single-shot imaging spectroscopy in timefrequency space using an echelon mirror, which overcomes the above difficulties. Using our scheme with the optical Kerr gate (OKG) apparatus, we successfully measured the time-frequency two-dimensional (2D) images of ultrashort laser pulses and therefore evaluated the full time-dependent intensity and phase of the laser pulses with the phase-retrieval procedure. By simply replacing the Kerr medium with actual samples, we could also map the phonon-polariton oscillations in ferroelectric $\mathrm{LiNbO}_{3}$. These experimental results show that our technique is useful not only for monitoring ultrashort laser pulses but also for investigating and coherently controlling the ultrafast dynamics of materials.

Figures 1(a) and 1(b) show the concept of our singleshot time-frequency imaging spectroscopy with an echelon mirror and the schematic experimental setup with the OKG apparatus, respectively. The echelon mirror is fabricated on a Ni block $\left(10 \times 10 \times 10 \mathrm{~mm}^{3}\right)$ with 500 steps in order to generate a spatially encoded time delay for the probe pulse. Each step has a step width of $20 \mu \mathrm{m}$ and a step height of $5 \mu \mathrm{m}$, which produce temporal steps of $\sim 34 \mathrm{fs}$ and an overall time delay of $\sim 17 \mathrm{ps}$. Here, the echelon mirror acts as a spatially encoded time delay optic but not as a spectral-resolved reflective grating. The detail of the echelon mirror is described elsewhere $[16,17]$.

As a light source, we used a conventional Ti:sapphire regenerative amplifier system with pulse duration, center wavelength, and repetition rate of $\sim 130 \mathrm{fs}, 800 \mathrm{~nm}$, and $1 \mathrm{kHz}$, respectively. The output of the regenerative amplifier system is divided into probe and gate pulses by a beam splitter (BS); the probe pulse is expanded to a diameter of $\sim 2 \mathrm{~cm}$ to cover a whole area of the echelon mirror with almost homogeneous intensity, while the gate pulse goes through an optical delay stage to tune the time delay between the probe and gate pulses. Subsequently, both beams are focused on a Kerr medium 
(a) Spectrometer

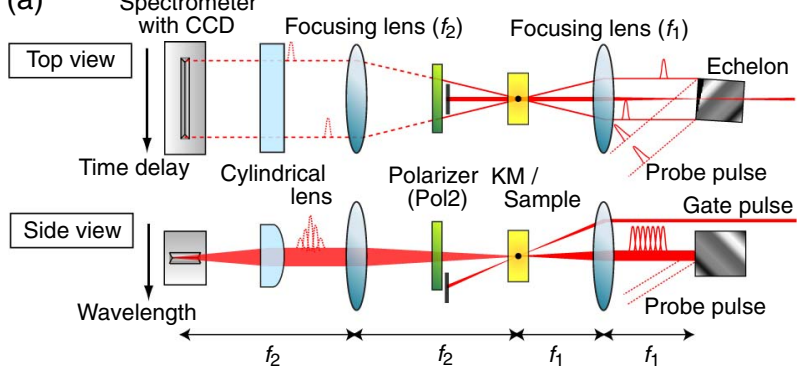

(b)

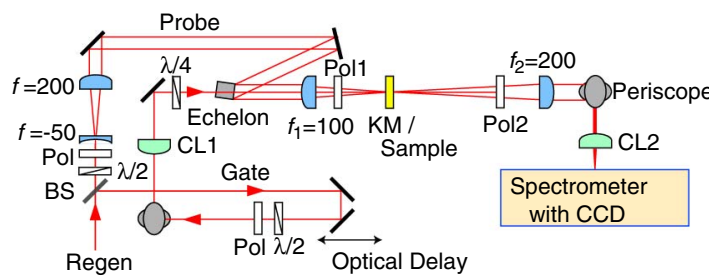

Fig. 1. (Color online) (a) Concept of single-shot timefrequency imaging spectroscopy with an echelon mirror and (b) schematic experimental arrangement. Regen, Ti:sapphire regenerative amplifier; $\mathrm{BS}$, beam splitter; $\lambda / 2, \lambda / 4$, half- and quarter-wave plates; CL, cylindrical lens; Pol, polarizer; KM, Kerr medium.

(KM) using a focusing lens $\left(f_{1}\right)$. The probe pulse is linearly polarized while the polarization of the gate is set to $45^{\circ}$ against that of the probe.

In the absence of the gate pulse, the probe pulse cannot be passed through the rest of the measurement apparatus due to a crossed second polarizer (Pol2), which is placed just after the Kerr medium. When the gate pulse is unblocked and then intersects with the probe pulse in a thin $\mathrm{KM}$ (quartz plate), the probe pulse can be passed through the crossed polarizer due to the optical Kerr effect. Because the thin, solid-state quartz plate works instantaneously as a Kerr shutter without molecular reorientations, the temporal resolution is basically limited by the pulse duration of the laser pulse itself [18]. The probe pulse transmitted in part through the crossed polarizer is therefore imaged linearly onto the entrance slit of a spectrometer with a 2D CCD using a focusing lens $\left(f_{2}\right)$ and a cylindrical lens (CL2). Consequently, in this manner, we are able to obtain time-frequency $2 \mathrm{D}$ images of the ultrashort laser pulses, that is, polarization-gating (PG) FROG traces of the laser pulses, on a single-shot basis.

Figure 2(a) shows the measured PG-FROG traces for negatively chirped (NC), nearly Fourier transfer-limited (TL), and positively chirped (PC) laser pulses. The chirp amount was tuned by moving the position of a built-in compressor of the regenerative amplifier. They can be reproduced well by their retrieved PG-FROG traces using FROG software (Femtosoft) within $0.5 \%$ error, as shown in Fig. 2(b). Note that, normally, the shorter-wavelength part of the laser pulse is passed earlier than the longerwavelength part in the NC pulse, but vice versa in the PC pulse. In our experimental apparatus, however, because the probe pulse has a spatially encoded time delay, the FROG images appear to be in the opposite direction; that is, the longer-wavelength part looks earlier than the shorter-wavelength part in the NC pulse, and vice versa in the PC pulse. (a) Measured

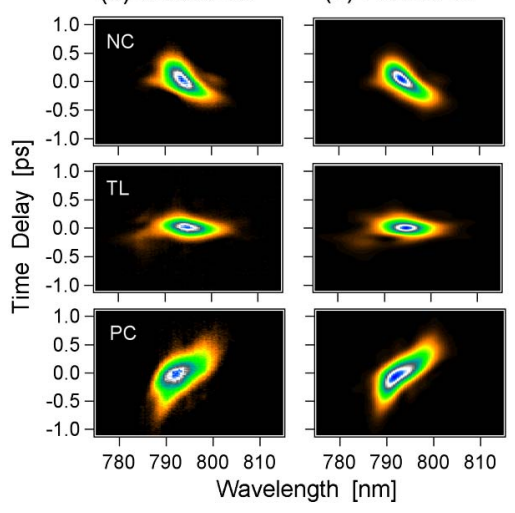

(c)

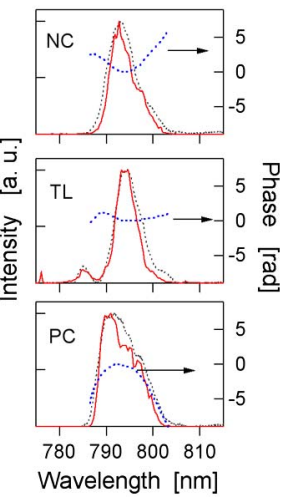

Fig. 2. (Color online) (a) Measured and (b) retrieved PGFROG traces for NC (top), nearly Fourier TL (middle), and PC (bottom) laser pulses. (c) Spectral profiles and phase shifts for the NC, nearly Fourier TL, and PC laser pulses.

Very recently, we successfully evaluated the intensity and phase profiles of ultrashort laser pulses using the second harmonic generation (SHG) FROG apparatus with an echelon mirror [16]. However, because of the nature of the second-order correlation between probe and gate pulses, the chirp direction of the laser pulse (either the PC or NC pulse) is not determined straightforwardly. On the other hand, using the PG-FROG apparatus shown here, the chirp direction can be simply evaluated from the FROG image due to its third-order correlation characteristic $[\underline{13}, 19]$. From the retrieved PG-FROG traces in Fig. 2(b), we could easily obtain the second- and thirdorder derivatives of the chirp profiles as $-1.39 \times 10^{4} \mathrm{fs}^{2}$ and $1.89 \times 10^{5} \mathrm{fs}^{3}$ for the NC pulses, $3.13 \times 10^{2} \mathrm{fs}^{2}$ and $5.16 \times 10^{5} \mathrm{fs}^{3}$ for the nearly Fourier TL pulses, and $2.35 \times$ $10^{4} \mathrm{fs}^{2}$ and $4.78 \times 10^{5} \mathrm{fs}^{3}$ for the PC pulses. These values were also numerically confirmed by the calculation from the geometrical configuration of the built-in compressor $[16,20]$. The value of the second-order derivative for the nearly Fourier TL pulse is 2 orders of magnitude smaller than those of the NC and PC pulses, although it is not ideally 0 .

The intensity and phase profiles of the $\mathrm{NC}$, nearly Fourier TL, and PC laser pulses obtained from the PG-FROG traces are shown in Fig. 2(c). The measured and retrieved spectral intensities are shown by dotted and solid curves, respectively. The retrieved phase shifts are also indicated by broken lines. The retrieved spectral intensity is in quite good agreement with the real power spectrum of the laser pulse. The phase shifts of the NC and PC pulses follow the quadratic dependence, reflecting the linear chirp of the laser pulses. On the other hand, the phase shift of the nearly Fourier TL is almost flat, as expected for the ideal Fourier TL pulses. These experimental results show that our PG-FROG apparatus using an echelon mirror works well to evaluate the characteristics of the amplified ultrashort laser pulses.

Next, we replaced the Kerr medium with a ferroelectric $x$-cut $\mathrm{LiNbO}_{3}$ thin plate with $0.5 \mathrm{~mm}$ thickness to demonstrate the ultrafast measurement of real samples using our new apparatus for single-shot imaging spectroscopy with an echelon mirror. In this configuration, the gate pulse acts as the pump pulse to induce the 

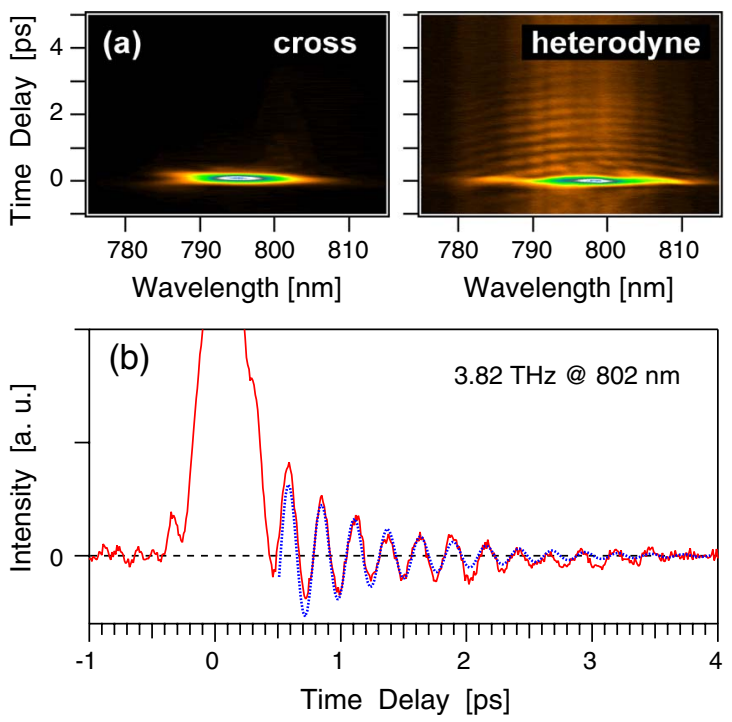

Fig. 3. (Color online) (a) Time-frequency images of ultrafast responses of ferroelectric $\mathrm{LiNbO}_{3}$ induced by the ISRS process. (b) Temporal profile of the E-mode phonon-polariton oscillations observed at $802 \mathrm{~nm}$ in the heterodyne detection. The dotted line shows the best fit for the observed dumped oscillations with a period of $3.82 \mathrm{THz}$ and a dephasing time of $1 \mathrm{ps}$.

asymmetric E-mode phonon polaritons in $\mathrm{LiNbO}_{3}$ via the impulsive stimulated Raman scattering (ISRS) processes $[21,22]$; that is, the polarization of the pump pulse is set parallel to the (101) direction, and that of the probe pulse is parallel to the ordinary axis (100) of the $\mathrm{LiNbO}_{3}$ thin plate.

Figure 3(a) shows the time-frequency 2D images of the ultrafast responses of the $\mathrm{LiNbO}_{3}$ thin plate induced by the ISRS process. When the second polarizer (Pol2) was completely crossed against the first polarizer (Pol1), we could only observe an instantaneous electronic response at the time origin. When the second polarizer was tilted a little bit from the completely crossed configuration (heterodyne detection), on the other hand, we could clearly observe the oscillations due to the E-mode phonon polaritons with continuous backgrounds coming from the reference pulses that survived through the second polarizer. The temporal profile of the oscillations observed at $802 \mathrm{~nm}$, which is reproduced by slicing off the observed 2D image, is also shown in Fig. 3(b). We found that the observed dumped oscillations have a period of $3.82 \mathrm{THz}$ and a dephasing time of $1.0 \mathrm{ps}$ as shown by a dotted line, whose values depend on the wavelength. The detailed experimental results will be discussed elsewhere.

In summary, we have proposed and demonstrated a new scheme for measuring the time-frequency $2 \mathrm{D}$ images of ultrashort laser pulses using an echelon mirror that is fabricated on a Ni block with 500 steps to generate a spatially encoded time delay for the probe pulse. By using the PG-FROG apparatus with an echelon mirror, the time-frequency 2D images of the ultrashort laser pulses with different chirp characteristics were successfully mapped on a single-shot basis. Consequently, the intensity and spectral phase of the laser pulses were eval- uated using the phase retrieval procedure. Because our technique has capability for direct measurements of molecular and solid-state dynamics by simply replacing the Kerr medium with samples, we could also observe the time-frequency 2D images of the phonon-polariton oscillations in ferroelectric $\mathrm{LiNbO}_{3}$ via the ISRS processes. Hence, we believe that this technique becomes a powerful spectroscopic tool not only for monitoring amplified ultrashort laser pulses but also for investigating and coherently controlling the ultrafast dynamics of materials.

We thank $\mathrm{H}$. Yamaki for his help in $\mathrm{LiNbO}_{3}$ experiments. This work was supported in part by Grants-inAid for Scientific Research (Nos. 22654034, 23104713, and 23241034) from the Japan Society for the Promotion of Science (JSPS) and the Ministry of Education, Culture, Sports, Science and Technology (MEXT) and the Nakatani Foundation of Electronic Measuring Technology Advancement. I. Katayama also acknowledges financial support for the Special Coordination Funds for the Japan Science and Technology Agency (JST).

\section{References}

1. P. Hannaford, ed., Femtosecond Laser Spectroscopy (Springer, 2005).

2. R. J. Gordon and Y. Fujimura, eds., Quantum Control of Molecular Reaction Dynamics (World Scientific, 2001).

3. D. B. Turner and K. A. Nelson, Nature 466, 1089 (2010).

4. K. Misawa and T. Kobayashi, J. Chem. Phys. 113, 7546 (2000).

5. P. R. Poulin and K. A. Nelson, Science 313, 1756 (2006).

6. G. P. Wakeham and K. A. Nelson, Opt. Lett. 25, 505 (2000).

7. N. Furukawa, C. E. Mair, V. D. Kleiman, and J. Takeda, Appl. Phys. Lett. 85, 4645 (2004).

8. Y. Makishima, N. Furukawa, A. Ishida, and J. Takeda, Jpn. J. Appl. Phys. 45, 5986 (2006).

9. Y. Paskover, I. Sh. Averbukh, and Y. Prior, Opt. Express 15, 1700 (2007).

10. K. Y. Kim, B. Yellampalle, A. J. Taylor, G. Rodriguez, and J. H. Glownia, Opt. Lett. 32, 1968 (2007).

11. C. Iaconis and I. A. Walmsley, Opt. Lett. 23, 792 (1998).

12. D. French, C. Dorrer, and I. Jovanovic, Opt. Lett. 34, 3415 (2009).

13. R. Trebino, Frequency-Resolved Optical Gating: The Measurement of Ultrafast Laser Pulses (Kluwer Academic, 2000).

14. K. W. Delong, R. Trebino, and D. J. Kane, J. Opt. Soc. Am. B 11, 1595 (1994).

15. D. J. Kane and R. Trebino, Opt. Lett. 18, 823 (1993).

16. I. Katayama, H. Sakaibara, and J. Takeda, Jpn. J. Appl. Phys. 50, 102701 (2011).

17. H. Sakaibara, I. Katayama, and J. Takeda, in Conference on Lasers and Electro-Optics, OSA Technical Digest (CD) (Optical Society of America, 2010), paper JThE83.

18. J. Takeda, K. Nakajima, S. Kurita, S. Tomimoto, S. Saito, and T. Suemoto, Phys. Rev. B 62, 10083 (2000).

19. A. M. Weiner, Ultrafast Optics (Wiley, 2009).

20. S. Backus, C. G. Durfee III, M. M. Murnane, and H. C. Kapteyn, Rev. Sci. Instrum. 69, 1207 (1998).

21. O. Albert, C. A. Gautier, J. C. Loulergue, and J. Etchepare, Solid State Commun. 107, 567 (1998).

22. C.-C. Lee, C.-T. Chia, Y.-M. Chang, M. L. Sun, and M.-L. Hu, Jpn. J. Appl. Phys. 43, 6829 (2004). 